\title{
Benchmarking of Java Verification Tools at the Software Verification Competition (SV-COMP)
}

\author{
Lucas Cordeiro \\ University of Manchester \\ Manchester, UK \\ lucas.cordeiro@ \\ manchester.ac.uk
}

\author{
Daniel Kroening \\ University of Oxford \\ Oxford, UK \\ kroening@cs.ox.ac.uk
}

\author{
Peter Schrammel \\ University of Sussex \\ Brighton, UK \\ p.schrammel@sussex.ac.uk
}

\begin{abstract}
Empirical evaluation of verification tools by benchmarking is a common method in software verification research. The Competition on Software Verification (SV-COMP) aims at standardization and reproducibility of benchmarking within the software verification community on an annual basis, through comparative evaluation of fully automatic software verifiers for C programs. Building upon this success, here we describe our proposal to re-use the ecosystem developed around SV-COMP for benchmarking Java verification tools. We provide a detailed description of the rules for benchmark verification tasks, the integration of new tools into SV-COMP's benchmarking framework and also give experimental results of a benchmarking run on three state-of-the-art Java verification tools, JPF-SE, JayHorn and JBMC.
\end{abstract}

\section{INTRODUCTION}

The complexity of software in smartphones and enterprise applications has dramatically increased over the last years. In particular, mobile applications based on the Android OS have gained popularity in the consumer electronics industry, reaching nearly $87 \%$ market share [1]; the Android OS essentially consists of a large set of libraries (approximately 13 million lines of code), containing both Java code and native code, which thus require methods to verify its security properties (e.g., sensitive data leakage) 2]. Similarly, Java remains popular in business applications (server-side), mainly owing to the existence of several robust frameworks (e.g., Spring $[3]$ ); therefore, verification of Java enterprise applications is also of particular interest.

Technology companies such as Facebook and Amazon increasingly invest effort and time to develop efficient and effective verification methods as testing alternatives 4, 5, to check correctness of some aspects of their systems with the goal to improve robustness and security 6. Although there are several software verification tools for Java programs (e.g., Bandera [7], JPF-SE [8], JayHorn [9] and JBMC [10]), they are typically very difficult to compare in practice, mainly due to the lack of (1) a common set of benchmarks and (2) methods to standardize and reproduce the empirical evaluations.

The Software Verification Competition. SV-COMP is one of the main initiatives targeted at the evaluation of new software verification methods, technologies, and tools [11. It has been running since 2012 as part of the International Conference on Tools and Algorithms for the Construction and Analysis of Systems (TACAS). Its main focus has been on evaluating different verification (and testing) tools for $\mathrm{C}$ programs. Currently, there are some powerful Java verifiers available, but there is no standard procedure to compare them fairly. One of the main difficulties to conduct such a comparison is the lack of a standard set of Java benchmarks and respective benchmarking infrastructure to obtain reliable, reproducible and accurate results.
The main contribution of this paper is to define a standard Java benchmark format and respective benchmarking infrastructure, which can drive the verification community to effectively evaluate state-of-the-art software verification tools for Java programs with the goal to achieve comparability and reproducibility. In particular, we collect and harmonize an initial set of Java benchmarks from different sources $9,10,12,13$ and re-use existing benchmarking infrastructure 14, so that we allow the community to get beyond research prototypes to usable tools [15. This can lead to further progress in the area of verification of Java programs and raise interest in applying these tools to industrial systems.

Java verification tools. Here, we consider the following tools:

JBMC $10{ }^{1}$ is based on the C Bounded Model Checker (CBMC) 16 to verify Java bytecode. JBMC consists of a frontend for parsing Java bytecode and a Java operational model (JOM), which is an exact but verification-friendly model of the standard Java libraries. A distinct feature of JBMC is the use of Bounded Model Checking (BMC) 17 in combination with Boolean Satisfiability and Satisfiability Modulo Theories (SMT) 18 and full symbolic state-space exploration, which allows JBMC to perform a bitaccurate verification of Java programs.

Java PathFinder (JPF) ${ }^{2}$ is an explicit-state 8 and symbolic 12 13 software model checker for Java bytecode. JPF is used to find and explain defects, collect runtime information as coverage metrics, deduce test vectors, and create corresponding test drivers for Java programs. JPF checks for property violations such as deadlocks or unhandled exceptions along all potential execution paths as well as user-specified assertions.

JayHorn ${ }^{3}$ is a verifier for Java bytecode 9 that uses the Java optimization framework Soot [19] as a front-end and then produces a set of constrained Horn clauses to encode the verification condition (VC). JayHorn is able to check for user-specified assertions and is sound for Java programs that use a single thread, have no dynamic class loading and complex static initializers.

Overview. This paper proposes a Java Category for the Software Verification Competition (SV-COMP). First, we describe in detail the definition and set up of the category. Then, we report on the integration of the three tools mentioned above into the SV-COMP benchmarking infrastructure. Finally, we give the experimental results that we obtained by benchmarking the tools on the benchmarks that we collected.

\footnotetext{
${ }^{1}$ Available at https://www.cprover.org/jbmc/

${ }^{2}$ Available at https://github.com/javapathfinder, https:// github.com/symbolicpathfinder

${ }^{3}$ Available at https://github.com/jayhorn/jayhorn
} 


\section{OUR PROPOSAL FOR A JAVA CATEGORY IN SV-COMP}

We describe our proposal to host a Java category in SV-COMP. In particular, we define the structure and meaning of verification tasks, the properties to be verified, the execution environment and how verification results could be evaluated 4

\subsection{Definition of Verification Task}

A verification task consists of a Java program and a specification. A verification run is a non-interactive execution of a competition candidate, i.e., a verifier, on a single verification task, in order to check whether the program satisfies its specification. According to the current SV-COMP rules 5 the result of a verification run is a triple (answer, witness, time). Answer is one of the following outcomes given in Table 1 .

Table 1: Definition of a Verification Result in SV-COMP 11.

\begin{tabular}{|l|l|}
\hline TRUE & $\begin{array}{l}\text { The specification is satisfied (i.e., there is no } \\
\text { path that violates the specification). }\end{array}$ \\
\hline FALSE & $\begin{array}{l}\text { The specification is violated (i.e., there exists } \\
\text { a path that violates the specification). }\end{array}$ \\
\hline UNKNOWN & $\begin{array}{l}\text { The tool cannot decide the problem or ter- } \\
\text { minates by a tool crash, time-out, or out-of- } \\
\text { memory (i.e., the competition candidate does } \\
\text { not succeed in computing an answer TRUE or } \\
\text { FALSE). }\end{array}$ \\
\hline
\end{tabular}

Time is the consumed CPU time until the verifier terminates. It includes the consumed CPU time of all processes that the verifier starts. If time is equal to or larger than the time limit, then the verifier is terminated and the answer is set to "timeout" (and interpreted as UNKNOWN).

Witness checking as previously described in 21]) represents an important feature to validate verification results given by verifiers. At the moment there is no witness checking in the Java category, though.

The Java verification tasks are partitioned into categories, which are defined in category-set files. At the moment there is only one category in Java, ReachSafety, which is concerned with specifications that consider an assert(condition) statement in the verification task whose non-violation must be proven or refuted.

\subsection{Benchmark Verification Tasks}

All Java verification tasks used in the competition must be part of the SV-COMP benchmark collection prior to the benchmark contribution deadline (typically in September). The competition candidates can "train", i.e., run and tune, their verifiers on the verification tasks until the tool submission deadline (typically in November). Misclassified benchmarks can be corrected during this training period. Contentious benchmarks are excluded from the competition once they are identified. SV-COMP does not use verification tasks without training; this is particularly important for Java since it has many features of which verifiers only support a subset; participants should know before the competition which features they are expected to support.

\footnotetext{
${ }^{4} \mathrm{SV}-\mathrm{COMP} 2019$ is in preparation at the time of publication. An up-to-date version of the rules can be found in 20].

${ }^{5} \mathrm{~A}$ detailed description of the current rules can be found in https: //sv-comp.sosy-lab.org/2018/rules.php

https://github.com/sosy-lab/sv-benchmarks
}

Benchmark structure. Verification tasks are grouped in directories, e.g., jbmc-regression. Within these directories, each verification task is in its own directory, e.g., StringValueOf01_true-assert. The suffix of this directory contains the expected verification outcome, i.e., _true-assert means that there is no execution of the program that violates any of the assertions in that program. A verification task has the directory structur ${ }^{7}$ shown in Table 2 target/ holds the build products if a verification tool has to compile the source code before performing its analysis. For convenience, a build script is provided with the benchmark.

Table 2: Directory structure of a verification task.

$\begin{array}{ll}\text { src } & \\ \text { src/main/java/Main.java } & \text { The Main class. } \\ \text { src/main/java/ } & \text { This directory contains the remain- } \\ & \text { ing Java source tree. } \\ \text { target/classes } & \text { After building, contains the .class } \\ & \text { files built from src. } \\ \text { target/target.jar } & \text { Assembles the .class files from tar- } \\ & \text { get/classes. }\end{array}$

The programs are assumed to be written in Java 1.8. Verification tools that require the sources to be compiled can use any Java 1.8 compiler. The .java source files are in the source tree in the src sub-directory of the benchmark directory. The program may call the Java standard library (java.*, javax.*). The sources of other dependencies must be added to the source tree together with their respective licenses. In order to allow tools that analyze Java source code to participate, we do not permit .jar files as dependencies (except the Java standard library). The benchmark must have a Main class with a public static void main(String[]) method in the root package. The Main.java file must have a copyright header indicating the source of the benchmark and its license.

Potential competition participants are invited to submit verification tasks until the benchmark contribution deadline by submitting a Pull Request to the benchmark collection repository 8 Verification tasks must comply with the aforementioned format. New proposed categories will be included if at least three different tools or teams participate in the category (i.e., not the same tool twice with a different configuration). In the following, we list a few conventions that are used in the Java verification tasks.

Assertions. For checking (un)reachability, we use the assert keyword provided in the Java language. It is assumed that the AssertionError thrown on violation of the assertion always leads to abortion of the program, i.e., it is not caught in the program. In future, further properties could be defined by different types of uncaught errors/exceptions.

Nondeterminism. The arguments to public static void main(String[] args) are assumed to be nondeterministic under the following constraints: assume $($ args $!=$ null $\& \&$ for all i. $0<=\mathrm{i}<$ args.length $=>$ $\operatorname{args}[i] !=$ null $)$.

We do not specify custom methods for introducing nondeterminism as done in the $\mathrm{C}$ categories of SV-COMP (cf. _ VERIFIER_nondet) 11. Instead, we use the java.util.Random class; the methods in that class are expected to return a nondeterministic value instead of a random value, but satisfying the same constraints on their value range.

\footnotetext{
${ }^{7}$ This structure is inspired by the Maven standard directory layout (cf. https://maven.apache.org).

https://github.com/sosy-lab/sv-benchmarks
} 


\begin{tabular}{|c|c|c|c|c|c|c|c|c|c|c|c|c|}
\hline Tool & \multicolumn{4}{|c|}{ JayHorn 0.5 .1} & \multicolumn{4}{|c|}{ JBMC 5.9} & \multicolumn{4}{|c|}{ JPF rev 32} \\
\hline Limits & \multicolumn{12}{|c|}{ timelimit: $900 \mathrm{~s}$, memlimit: $15000 \mathrm{MB}, \mathrm{CPU}$ core limit: 8} \\
\hline Host & \multicolumn{12}{|c|}{ localhost } \\
\hline OS & \multicolumn{12}{|c|}{ Linux 4.4.0-131-generic x86_64 } \\
\hline System & \multicolumn{12}{|c|}{ CPU: Intel Core i7-6700HQ CPU @ 2.60GHz with 8 cores, frequency: 3500 MHz, Turbo Boost enabled; RAM: 16014020 kB } \\
\hline Date of execution & \multicolumn{4}{|c|}{ 2018-07-29 02:53:52 BST } & \multicolumn{4}{|c|}{ 2018-07-28 18:40:30 BST } & \multicolumn{4}{|c|}{ 2018-07-29 16:02:12 BST } \\
\hline Run set & \multicolumn{4}{|c|}{ jayhorn.sv-comp18 } & \multicolumn{4}{|c|}{ jbmc.sv-comp18 } & \multicolumn{4}{|c|}{ jpf.sv-comp18 } \\
\hline ../git-sv-benchmarks/java/ & status & cputime & walltime & memUsage & status & cputime & walltime & memUsage & status & cputime & walltime & memUsage \\
\hline jbmc-regression/ArithmeticException1_false-assert & true & $2.895 \mathrm{~s}$ & $1.657 \mathrm{~s}$ & 195948544 & false(reach) & $0.260 \mathrm{~s}$ & 0.2735 & 43819008 & false(reach) & 1.0115 & 0.5955 & 67473408 \\
\hline jbmc-regression/ArithmeticException5_true-assert & true & 2.3085 & 1.3455 & 152911872 & true & 0.3465 & $0.365 \mathrm{~s}$ & 43417600 & false(reach) & 0.9985 & 0.6125 & 66256896 \\
\hline jbmc-regression/ArithmeticException6_false-assert & true & 2.3455 & 1.3465 & 150769664 & false(reach) & 0.3045 & 0.3285 & 43786240 & true & 0.9385 & 0.5845 & 64716800 \\
\hline jbmc-regression/ArrayIndexOutofBoundsException1_false-assert & false(reach) & $4.108 \mathrm{~s}$ & 2.3005 & 197890048 & false(reach) & $0.297 \mathrm{~s}$ & 0.3085 & 44023808 & true & 1.0115 & 0.6015 & 66256896 \\
\hline jbmc-regression/ArrayIndex0ut0fBoundsException2_false-assert & false(reach) & $4.161 \mathrm{~s}$ & 2.2965 & 193990656 & false(reach) & 0.3015 & 0.3165 & 43749376 & true & 0.9795 & $0.590 \mathrm{~s}$ & 63488000 \\
\hline jbmc-regression/ArrayIndex0ut0fBoundsException3_false-assert & false(reach) & 5.1375 & $2.827 \mathrm{~s}$ & 230653952 & false(reach) & 0.3115 & 0.3245 & 43880448 & true & 0.9185 & $0.577 \mathrm{~s}$ & 64274432 \\
\hline jbmc-regression/BufferedReaderReadLine_false-assert & false(reach) & 7.0105 & $3.658 \mathrm{~s}$ & 260804608 & false(reach) & $0.439 \mathrm{~s}$ & $0.447 \mathrm{~s}$ & 44457984 & true & 1.0065 & $0.616 \mathrm{~s}$ & 67321856 \\
\hline jbmc-regression/CharSequenceBug_false-assert & false(reach) & 4.4335 & 2.4165 & 192462848 & false(reach) & 0.3875 & 0.4025 & 44269568 & true & 0.9595 & $0.597 \mathrm{~s}$ & 63365120 \\
\hline jbmc-regression/CharSequenceToString_true-assert & false(reach) & 4.5155 & 2.4935 & 193818624 & true & 0.7125 & 0.7315 & 44658688 & true & 0.9705 & $0.580 \mathrm{~s}$ & 63336448 \\
\hline jbmc-regression/ClassCastException1_false-assert & false(reach) & $3.961 \mathrm{~s}$ & 2.2155 & 194396160 & false(reach) & 0.3285 & $0.335 \mathrm{~s}$ & 44134400 & false(reach) & $1.000 \mathrm{~s}$ & 0.6045 & 64888832 \\
\hline jbmc-regression/ClassCastException2_true-assert & true & $4.357 \mathrm{~s}$ & 2.3565 & 189190144 & true & 0.4425 & 0.4565 & 44097536 & true & 0.9505 & 0.5815 & 64630784 \\
\hline jbmc-regression/ClassCastException__false-assert & false(reach) & $3.969 \mathrm{~s}$ & 2.1865 & 192733184 & false(reach) & $0.297 \mathrm{~s}$ & $0.316 \mathrm{~s}$ & 43929600 & false(reach) & 0.9505 & 0.5845 & 64626688 \\
\hline jbmc-regression/Class_method1_true-assert & true & $3.129 \mathrm{~s}$ & $1.754 \mathrm{~s}$ & 184451072 & true & $0.467 \mathrm{~s}$ & 0.4865 & 43941888 & true & 0.9205 & $0.568 \mathrm{~s}$ & 63279104 \\
\hline jbmc-regression/Inheritancel_true-assert & true & 5.0235 & $2.652 \mathrm{~s}$ & 217104384 & true & $0.459 \mathrm{~s}$ & $0.471 \mathrm{~s}$ & 44077056 & true & $0.950 \mathrm{~s}$ & $0.578 \mathrm{~s}$ & 64221184 \\
\hline jbmc-regression/NegativeArraySizeException1_false-assert & true & $2.399 \mathrm{~s}$ & 1.3945 & 153817088 & false(reach) & 0.2915 & 0.3035 & 43745280 & false(reach) & 0.9615 & $0.578 \mathrm{~s}$ & 64442368 \\
\hline jbmc-regression/NegativeArraySizeException2_false-assert & true & $2.205 \mathrm{~s}$ & 1.3055 & 153120768 & false(reach) & 0.2855 & 0.3125 & 43921408 & false(reach) & $0.985 \mathrm{~s}$ & 0.6055 & 63938560 \\
\hline jbmc-regression/NullPointerException1_true-assert & unknown & $1.303 \mathrm{~s}$ & $0.761 \mathrm{~s}$ & 116211712 & true & 0.4315 & 0.4415 & 43900928 & false(reach) & $0.952 \mathrm{~s}$ & 0.5955 & 63512576 \\
\hline jbmc-regression/NullPointerException2_false-assert & unknown & $1.273 \mathrm{~s}$ & 0.7365 & 115384320 & false(reach) & 0.2865 & 0.2945 & 43929600 & false(reach) & 0.9495 & 0.5755 & 64266240 \\
\hline jbmc-regression/NullPointerException3_false-assert & unknown & 1.3255 & 0.7525 & 115838976 & false(reach) & 0.2665 & $\theta .275 \mathrm{~s}$ & 43737088 & false(reach) & 0.9735 & 0.5745 & 63655936 \\
\hline jbmc-regression/NullPointerException4_false-assert & unknown & $1.218 \mathrm{~s}$ & 0.7005 & 114372608 & false(reach) & 0.2825 & 0.2965 & 43929600 & false(reach) & 1.0025 & $0.613 \mathrm{~s}$ & 64077824 \\
\hline jbmc-regression/RegexMatches 01 true-assert & false(reach) & 5.2225 & 2.8145 & 195457024 & false(reach) & 38.2745 & 38.2885 & 237780992 & true & 1.0015 & $0.620 \mathrm{~s}$ & 63860736 \\
\hline jbmc-regression/Regex/Matches 02 false-assert & false(reach) & 6.1265 & 3.2355 & 218169344 & false(reach) & $38.763 \mathrm{~s}$ & $38.780 \mathrm{~s}$ & 243040256 & true & 0.9745 & $0.603 \mathrm{~s}$ & 63315968 \\
\hline jbmc-regression/RegexSubstitutione1_true-assert & false(reach) & 10.2115 & 5.2995 & 412655616 & true & 4.5375 & 4.5545 & 117723136 & true & 1.0385 & $0.645 \mathrm{~s}$ & 65220608 \\
\hline jbmc-regression/RegexSubstitution02_false-assert & false(reach) & $9.948 \mathrm{~s}$ & 5.2005 & 384372736 & true & 4.5925 & 4.6125 & 89309184 & true & 0.9785 & 0.5985 & 64528384 \\
\hline jbmc-regression/RegexSubstitution03_true-assert & false(reach) & $7.912 \mathrm{~s}$ & 4.1625 & 297971712 & true & $1.741 \mathrm{~s}$ & $1.765 \mathrm{~s}$ & 49369088 & true & $1.068 \mathrm{~s}$ & $0.638 \mathrm{~s}$ & 65187840 \\
\hline jbmc-regression/StaticCharMethods 01 true-assert & false(reach) & 4.9885 & 2.6045 & 233947136 & true & 0.9445 & 0.9665 & 46419968 & true & 0.9665 & $0.579 \mathrm{~s}$ & 62652416 \\
\hline jbmc-regression/StaticCharMethods 02 _false-assert & false(reach) & 5.0325 & $2.683 \mathrm{~s}$ & 0714624 & false(reach) & $1.094 \mathrm{~s}$ & 1.1045 & 53043200 & true & $0.960 \mathrm{~s}$ & $0.585 \mathrm{~s}$ & 63422464 \\
\hline jbmc-regression/StaticCharMethods03_false-assert & false(reach) & $4.816 \mathrm{~s}$ & 2.6225 & 198123520 & false(reach) & $1.084 \mathrm{~s}$ & $1.096 \mathrm{~s}$ & 51294208 & true & 1.0225 & 0.6325 & 64135168 \\
\hline jbmc-regression/StaticCharMethods 04 false-assert & false(reach) & $4.843 \mathrm{~s}$ & 2.6365 & 200192000 & false(reach) & 1.0465 & $1.055 \mathrm{~s}$ & 51044352 & true & 0.9065 & 0.5515 & 63311872 \\
\hline jbmc-regression/StaticCharMethods 05 false-assert & false(reach) & 7.8225 & $4.077 \mathrm{~s}$ & 278511616 & false(reach) & 1.8245 & 1.8335 & 59617280 & unknown & $0.986 \mathrm{~s}$ & 0.5985 & 60248064 \\
\hline jbmc-regression/StaticCharMethods06_true-assert & false(reach) & 6.2725 & 3.2715 & 37772800 & true & 2.6875 & 2.6975 & 53399552 & true & 1.0085 & 0.6245 & 64339968 \\
\hline jbmc-regression/StringBuilderAppende1_true-assert & fralse(reach) & $15.775 \mathrm{~s}$ & 8.0965 & 521629696 & unknown & 874.9365 & 875.0355 & 63283200 & true & 1.0085 & $0.610 \mathrm{~s}$ & 65589248 \\
\hline jbmc-regression/StringBuilderAppende2 false-assert & false(reach) & $16.517 \mathrm{~s}$ & 8.5405 & 539189248 & unknown & 874.9625 & 875.0305 & 129449984 & true & $1.007 \mathrm{~s}$ & 0.6165 & 63279104 \\
\hline
\end{tabular}

Figure 1: Per benchmark comparison table as produced by BenchExec [14]. The detailed description of the scores of each tool can be found in https://pschrammel.bitbucket.io/schrammel-it/research/sv-comp-java-2018/

Moreover, we do not specify a custom assume method. It is recommended to use Runtime.getRuntime().halt(1) to achieve the desired behavior as this does not impact the termination behavior of a program - e.g. defining assume(cond); as while(!cond); would make any program with such assumptions classified non-terminating when a potential Java Termination category might be introduced in future.

Operating System Model. Any library methods that make system calls are not allowed in verification tasks. Exceptions with well-defined behaviors can be explicitly granted if they allow a wider range of benchmarks to be included in the collection. For instance, new java.util.Date() could be defined to create a Date object with a nondeterministic timestamp.

\subsection{Properties}

In SV-COMP, the specification to be verified for a program path/ dirname is given either in a file with the name path/dirname.prp or in a file Category.prp. The definitions in these .prp have been designed for extensibility in order to allow new properties for future categories to be specified. For instance, in the $\mathrm{C}$ categories, four different properties are in use.

For our Java category, the definition in ReachSafety.prp states CHECK( init(Main.main), LTL(G assert) ). Here, init(Main.main) gives the initial states of the program by a call of function main (under the assumptions on the inputs of main stated in the previous section). $L T L(f)$ specifies that formula $f$ holds at every initial state of the program. In particular, the linear-time temporal logic (LTL) operator $\mathrm{G} f$ means that $f$ globally holds. The proposition assert is true if all assert statements in the program hold.

\subsection{Competition Environment and Requirements}

In the SV-COMP environment, each software verifier is assumed to run on a machine with a GNU/Linux operating system (x86_64linux, Ubuntu 16.04). SV-COMP also sets three resource limits to evaluate each software verifier, which are: $(i)$ a memory limit of 15 GB of RAM, (ii) a runtime limit of 900 seconds of CPU time, and (iii) a limit to 8 processing units of a CPU. Note that if a software verifier does not consume CPU time, then it is killed after 900 seconds of wall clock time, and the resulting runtime is set to 900 seconds. For the Java category, OpenJDK 1.8 is assumed to be installed on the competition machines. The modest resource requirements have been chosen in order to allow everybody to reproduce the competition results on a reasonably sized machine.

\subsection{Evaluation by Scores and Runtime}

SV-COMP has strict rules to evaluate the verification results provided by each software verifier. In particular, each verifier is heavily penalized if they produce an incorrect result for a specific verification task with the goal to favor correctness. The scores are assigned to each software verifier according to Table $3^{9}$

The higher score and penalty for the TRUE case is justified because it is usually more difficult to prove a program correct than to find a bug 11 .

\section{INTEGRATION INTO THE COMPETITION INFRASTRUCTURE}

BenchExed 14 is the framework used in SV-COMP for reliable benchmarking and resource measurement. It can be easily in-

https://sv-comp.sosy-lab.org/2018/rules.php

10 https://github.com/sosy-lab/benchexec 




Figure 2: Example of a scatter plot comparing JPF-SE and JBMC as produced by BenchExec 22.

Table 3: Evaluation by Scores and Runtime in SV-COMP 11.

\begin{tabular}{|l|l|l|}
\hline Points & Answer & Description \\
\hline 0 & UNKNOWN & $\begin{array}{l}\text { Failure to compute verification re- } \\
\text { sult, out of resources, program crash. }\end{array}$ \\
\hline+1 & $\begin{array}{l}\text { FALSE } \\
\text { correct }\end{array}$ & The error in the program was found. \\
\hline-16 & $\begin{array}{l}\text { FALSE } \\
\text { incorrect }\end{array}$ & $\begin{array}{l}\text { An error is reported for a program } \\
\text { that fulfills the specification (false } \\
\text { alarm, incomplete analysis). }\end{array}$ \\
\hline+2 & $\begin{array}{l}\text { TRUE } \\
\text { correct }\end{array}$ & $\begin{array}{l}\text { The program was analyzed to be free } \\
\text { of errors. }\end{array}$ \\
\hline-32 & $\begin{array}{l}\text { TRUE } \\
\text { incorrect }\end{array}$ & $\begin{array}{l}\text { The program had an error but the } \\
\text { competition candidate did not find } \\
\text { it (missed bug, unsound analysis). }\end{array}$ \\
\hline
\end{tabular}

stalled to run experimental comparisons and to reproduce competition results. For the Java category, we extended the framework by introducing a new assert proposition for specifying properties.

Integrating a new tool into the framework requires the addition of two files:

- The tool-info module is a Python module located in the benchexec/tools directory that implements the tool interface to connect a verifier to BenchExec. Essentially, it must provide functions for running the verifier with a given verification task and to translate the tool output into an answer TRUE, FALSE or UNKNOWN (see Section 2.1).

- The benchmark definition ${ }^{11}$ is an XML file that specifies which categories can be run with a given verifier and which tool command line options to use.

Here, we have implemented and added these files for the three tools under consideration (i.e., JPF-SE, JBMC and JayHorn). After installing a verifier (e.g., JPF-SE) in the base directory of BenchExec, it can be run with the command bin/benchexec jpf.xml. There are various options to run subsets of the verification tasks and overriding time and memory limits, for instance.

$\overline{11} \overline{\text { https://github.com/sosy-lab/sv-comp }}$

\section{JAVA BENCHMARK COLLECTION}

Previously, there existed 64 "minepump" benchmarks in the SVCOMP repository from earlier attempts to run a Java category; these benchmarks were already classified as "safe" and "unsafe" by the community. Beyond these few files, there was no standard benchmark suite for Java verification available in the community ${ }^{12}$ Therefore, we took the entire JBMC regression test suite ("jbmc-regression"), consisting of 177 benchmarks (including known bugs and hard benchmarks that JBMC cannot yet handle); these benchmarks test common Java features (e.g., polymorphism, exceptions, arrays, and strings) and they were classified by the JBMC developers. We also used 23 benchmarks ("jayhornrecursive") taken from the JayHorn repository 9. These are mainly $\mathrm{C}$ benchmarks from the recursive category that have been translated into Java by keeping the original classification from SVCOMP. Additionally, we have extracted 104 benchmarks from the JPF regression test suite 12 ("jpf-regression"); for these particular benchmarks, we have manually inspected and classified them as "safe" and "unsafe". Table 4 summarizes the characteristics of the benchmark sets.

Table 4: Characteristics of the Java Benchmark Sets.

\begin{tabular}{lrrrr}
\hline benchmark set & total & safe & unsafe & avg. LOC \\
\hline jbmc-regression & 177 & 89 & 88 & 25 \\
jpf-regression & 104 & 52 & 52 & 52 \\
jayhorn-recursive & 23 & 14 & 9 & 35 \\
minepump & 64 & 8 & 56 & 62 \\
\hline total & 368 & 163 & 205 & 40 \\
\hline
\end{tabular}

These benchmarks are a good start to launch the proposed Java category, but are not yet fully representative for the breadth of challenges that we face in verifying Java programs. We will rely on the community to contribute and continuously enrich the collection of Java benchmarks in the future.

\section{BENCHMARKING RESULTS}

The results of running a verifier using BenchExec as explained in Section 3 are collected in a timestamped format in the results directory with the BenchExec base directory. This contains a .zip file with the $\log$ files and a .xml.bz2 file with the results in a structured format. One or more of the latter files (potentially of different tools) can be passed to bin/table-generator in order to generate an HTML report that compares the benchmarking runs.

A part of this report is shown in Figure $1{ }^{13}$ This comparison used JBMC v5.9-3c2e55e, JayHorn v0.5.1, and JPF-SE rev 32. The HTML report allows to filter rows and columns and display the most important comparison charts, such as scatter plots Figure 2 compares the CPU time of JPF-SE and JBMC, for example - and quantile ("cactus") plots as depicted in Figure 3 The latter plot shows the cumulative time (y-axis) required for a verifier to solve its $n$ fastest benchmarks (x-axis). This allows us to compare the scaling behavior of the tools, i.e., the longer a graph extends to the right the more verification tasks were solved by the tool, the closer to the bottom the faster it is.

\section{CONCLUSIONS}

We described our proposal to run the first Java category in SVCOMP, given that it is currently focused on evaluating $\mathrm{C}$ software verifiers only. In particular, we defined the structure and meaning of verification tasks, the properties to be verified, the execution environment and how verifiers are integrated into the

${ }^{12}$ There is a community effort in collecting Java benchmarks in http://sir.unl.edu, but they are not currently classified.

${ }^{13}$ The full results are available at https://pschrammel. bitbucket.io/schrammel-it/research/sv-comp-java-2018/ 


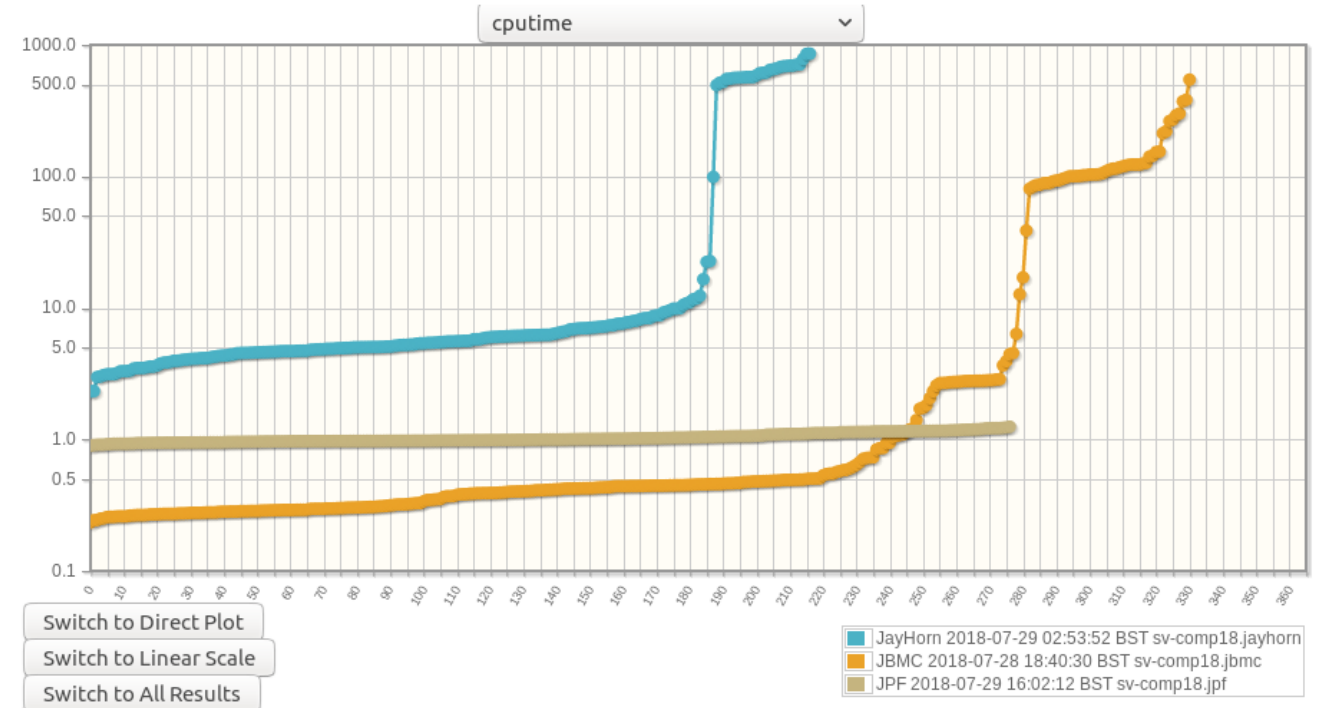

Figure 3: Quantile plot as produced by BenchExec 14 .

benchmarking framework and how verification results, produced by each Java verifier are evaluated. SV-COMP is one of the most successful software verification competitions, which is annually held by TACAS. Although the first edition of SV-COMP took place in 2012 and has been a successful so far, there has been no verification track to evaluate software verifiers targeted for Java programs. As a next step, Java verifiers need to be extended in order to produce witness files (for violation and correctness) that adhere to the witness exchange format defined by SV-COMP [21]. In this respect, witness checkers for Java verifiers also need to developed in order to check validity of the verification results provided by each verifier 23 .

\section{REFERENCES}

[1] IDC: Smartphone OS Market Share, 2016 Q3. http://www. idc.com/prodserv/smartphone-os-market-share.jsp Accessed 2018-07-29

[2] Bai, G., Ye, Q., Wu, Y., Botha, H., Sun, J., Liu, Y., Dong, J.S., Visser, W.: Towards model checking Android applications. IEEE Trans. Software Eng. 44(6), 595-612 (2018)

[3] Gupta, K.: Why Is Spring More Popular than Other Java Frameworks? https://www.freelancinggig.com/blog/ 2018/04/26/spring-popular-java-frameworks/Accessed 2018-07-29

[4] O'Hearn, P.W.: Continuous reasoning: Scaling the impact of formal methods. In: LICS, pp. 13-25 (2018)

[5] Cook, B., Khazem, K., Kroening, D., Tasiran, S., Tautschnig, M., Tuttle, M.R.: Model checking boot code from AWS data centers. In: CAV. LNCS, vol. 10982, pp. 467-486 (2018)

[6] Durumeric, Z., Kasten, J., Adrian, D., Halderman, J.A., Bailey, M., Li, F., Weaver, N., Amann, J., Beekman, J., Payer, M., Paxson, V.: The matter of Heartbleed. In: IMC, pp. $475-488$ (2014)

[7] Iosif, R., Dwyer, M.B., Hatcliff, J.: Translating java for multiple model checkers: The bandera back-end. Formal Methods in System Design 26(2), 137-180 (2005). doi:10.1007/s10703-005-1491-3

[8] Visser, W., Havelund, K., Brat, G.P., Park, S.: Model checking programs. In: ASE, pp. 3-12 (2000)

[9] Kahsai, T., Rümmer, P., Sanchez, H., Schäf, M.: JayHorn: A framework for verifying Java programs. In: CAV. LNCS, vol. 9779 , pp. 352-358 (2016)
[10] Cordeiro, L.C., Kesseli, P., Kroening, D., Schrammel, P., Trtík, M.: JBMC: A bounded model checking tool for verifying Java bytecode. In: CAV. LNCS, vol. 10981, pp. 183-190 (2018)

[11] Beyer, D.: Software verification with validation of results (report on SV-COMP 2017). In: TACAS. LNCS, vol. 10206, pp. 331-349 (2017)

[12] Anand, S., Pasareanu, C.S., Visser, W.: JPF-SE: A symbolic execution extension to Java PathFinder. In: TACAS. LNCS, vol. 4424, pp. 134-138 (2007)

[13] Pasareanu, C.S., Rungta, N.: Symbolic PathFinder: symbolic execution of Java bytecode. In: ASE, pp. 179-180 (2010)

[14] Beyer, D., Löwe, S., Wendler, P.: Reliable benchmarking: requirements and solutions. International Journal on Software Tools for Technology Transfer (to appear) (2017)

[15] Alglave, J., Donaldson, A.F., Kroening, D., Tautschnig, M.: Making software verification tools really work. In: ATVA. LNCS, vol. 6996, pp. 28-42 (2011)

[16] Clarke, E.M., Kroening, D., Lerda, F.: A tool for checking ANSI-C programs. In: TACAS. LNCS, vol. 2988, pp. 168-176 (2004)

[17] Biere, A.: Bounded Model Checking. Frontiers in Artificial Intelligence and Applications, vol. 185, pp. 457-481 (2009)

[18] Barrett, C., Sebastiani, R., Seshia, S.A., Tinelli, C.: Satisfiability Modulo Theories. Frontiers in Artificial Intelligence and Applications, vol. 185, pp. 825-885 (2009)

[19] Vallée-Rai, R., Co, P., Gagnon, E., Hendren, L., Lam, P., Sundaresan, V.: Soot - a Java bytecode optimization framework. In: CASCON, p. 13 (1999)

[20] Cordeiro, L., Kroening, D., Schrammel, P.: Benchmarking of Java Verification Tools at the Software Verification Competition (SV-COMP) (2018). http://arxiv.org/abs/1809.03739

[21] Beyer, D., Dangl, M., Dietsch, D., Heizmann, M.: Correctness witnesses: exchanging verification results between verifiers. In: FSE, pp. 326-337 (2016)

[22] Beyer, D., Löwe, S., Wendler, P.: Benchmarking and resource measurement. In: SPIN. LNCS, vol. 9232, pp. 160-178 (2015)

[23] Beyer, D., Dangl, M., Lemberger, T., Tautschnig, M.: Tests from witnesses - execution-based validation of verification results. In: TAP. LNCS, vol. 10889, pp. 3-23 (2018) 